Biannual Research Journal Grassroots

Vol.55, No.II, 2021: 103-119

Grassroots

\title{
PREVALENCE OF DEPRESSION AND CORRELATION BETWEEN FACTORS OF DEPRESSION IN TRAINEE LADY HEALTH VISITORS OF PAKISTAN: USING BDI-II
}

\author{
Nausheen Shahzad \\ PhD Fellow, Department of Psychology,University of Karachi @, Karachi \\ Email: nausheenshahzad0@gmail.com \\ Dr Anjum Ara \\ Professor Department of Psychology,University of Karachi@ Karachi
}

\begin{abstract}
Trainee lady health visitors in Pakistan have extensive problems in their daily fieldwork: sexual harassment, character calling, rude behavior of people, irregular wages and life-threatening situations (Diwan, 2013). TLHVs experience vulnerability and harassment by religious fundamentalists (Naqvi \& Khan, 2005). Their gender, age, socioeconomic background and unhealthy patterns of Pakistan's patriarchal society add more stress (Tabassum, 2016). Stress may cause depression therefore this study is conducted to explore the prevalence of depression in TLHVS of Karachi, Pakistan and to find the correlation of depression with somaticlaffective factor and cognitive factor of depression by using Beck Depression Inventory-II based on descriptive and correlational design. The results indicate the prevalence of moderate depression among TLHVs, further it indicates stronger positive correlation between depression and somatic/affective factors of depression than cognitive factors of depression.
\end{abstract}

Keywords: Trainee lady health visitors (TLHV), Cognitive symptoms, Somatic/Affective symptoms, Depression, Beck Depression Inventory (BDI-II)

\section{INTRODUCTION}

Trainee lady health visitors in Pakistan: Pakistan is situated in southern Asia along the coastline of the Arabian Sea. Karachi is the biggest city of Pakistan, located in the Province of Sindh, Karachi is often called mini-Pakistan and is the most heterogeneous area of the country with all ethnicities residing. This ethnic mix is the typical culture of Karachi. The city serves as a space where all the cultures are exhibited together. Karachi being the industrial hub attracts a large number of labor from all over country, labor has come and dwelled in 
slum areas of their own ethnic origin thus creating largest slums of Pakistan with $60 \%$ population living in slums in Karachi. Most of the girls in the slum areas, however, get themselves better educated than the boys of the slum areas (Hasan, 2010) however due to lack of monetary resources they can't be enrolled into colleges and universities so they prefer LHV programs which are free of cost and provide job security. Slums need health facilities too, which are fulfilled by the lady health visitors (Hasan, 2010) who are resident of the same community where they work for so that they understand the cultural, linguistic and ethnic background of that area (Peers for Progress, n.d.). Lady health visitors are a special cadre of ministry of health which started their noble cause in 1994 when the National Program for Family Planning and Primary Health Care was launched. There are 125,000 lady health workers in Pakistan, with 24,000 in Sindh at present. The main objective of the program is to provide awareness, preventive methods and cures at the community level (Planning Commission Government of Pakistan, 1994). Women were recruited, hired and trained to provide family planning and primary health care (Hafeez, Mohamud.,Shiekh, Shah \& Jooma, 2011). Trainee lady health visitors address child health care services, maternal services, antenatal care and chronic diseases treatment. Their job duties include providing education about birth control, family planning, safe pause between two births, as well as running polio campaigns, vaccination programs for infants and children, pandemic, and epidemic control (Planning Commission Government of Pakistan, 1994).

Problems Faced by Trainee lady health visitors of Pakistan: Demographic and epidemiological researches reveal that majority of the TLHVs belong to lower socioeconomic background, they live in ethnic slums where particular standards prevail regarding women's education, status in society, role and job selection. Pakistan is a male dominated society in which a woman is considered inferior to men due to religio-political doctrines and she is marginalized and segregated in society: a young woman is subjugated to these treatments more often than an elderly female or child (Tabassum, 2016). This is enough breeding environment for low self-esteem, helplessness, hopelessness, frustration, anger and stress. Trainee lady health visitors face many problems during the course of their work: sexual harassment, irregular 
wages, job insecurity, unstructured work description, uncertain working conditions, verbal abuse and unhealthy patterns of patriarchal society (Diwan, 2013). Health visitors experience vulnerability and sexual harassment by religious fundamentalists (Naqvi \& Khan, 2005). Trainee lady health visitors, in general, harbor concerns regarding removal or isolation from employment. It has also been found that they face verbal attacks and abuse by families that do not desire to vaccinate their child (Tsaras et.al., 2018).

Depression: Depression first appeared in written history in second millennium B.C.E in Mesopotamia. Depression was considered a spiritual problem caused by demons and treated by priests (Reynolds \& Wilson, 2013). Later on the Greek physician, Hippocrates coined the term melancholia for depression which was due to the excess of four body fluids called yellow bile, black bile, phlegm, and blood. He specifically believed that it was caused by black bile in the spleen and treated it with bloodletting baths, exercise and diet (Tipton, 2014). In the common era very harsh treatments were recommended for depression which included starvation and beating. Twentieth century treatments for depression started with the psychoanalytic approach, behavioral approach and cognitive approach.

The psychoanalytic approach by Sigmund Freud helped the depressed person by resolving his unconscious conflicts reducing selfdestructive thoughts and behavior (De Sousa, 2011). The behaviorist movement suggested that depression is a learned behavior and can also be unlearned through principals of learning such as association and reinforcement. Depressed people consider themselves helpless and inadequate. Later on Martin Seligman suggested the role of the learned helplessness in the development of depression (Pluskota, 2014). During the 1970s the medical model explained that all mental disorders are basically due to physiological factors and they focused on causes of depression on factors which included genetics, brain chemistry, hormones, and brain anatomy. A considerable extent of neuropsychological impairment remains after the mood symptoms resolution, at least in a significant extent of patients, irrespective of the affected domains (Porter et.al., 2016). The common neuropsychiatric syndromes among individuals include depression disorders, with an occurrence ranging from $26 \%$ to $65 \%$ (Chen et.al., 2016). After this explanation the use of anti-depressed medications became a popular 
treatment for depression. The term major depressive disorder (MDD) appeared in the 1970s and became a part of DSM-III in 1980.

Aaron Beck during the 1970's came up with cognitive theories of depression and explained that negative automatic thoughts, negative self-beliefs and errors in processing information are responsible for depressive symptoms. Beck argues that depression has three factors including somatic, cognitive and affective which help in diagnosing the presence of depression in a person. He developed a rating instrument called BDI to adequately understand the presence of depression along with its different factors which helps in treatment plans (Pluskota, 2014; Beck, Steer, \& Brown, 1996).

Depression was considered as a common disorder. A major challenge for clinicians is to not merely just treat depression but also to prevent relapse when it occurs (Sadler et.al., 2018). According to a study, depression has been documented to increase to second rank in both males and females. It has been estimated that approximately 10 44 percent of the individuals living in developing countries are influenced by depression disorders and approximately 50.8 million are undergoing depression (Mumtaz et.al., 2013). Schuch et.al., (2014) reported that the occurrence of major depressive disorders vary among genders. It is observed that women are more exposed to major depressive disorders than men and this is a finely developed concept within both clinical and community samples. Due to this variation, a lot of queries have evolved, related to the dual effect of major depressive disorders upon gender, working in the medical field. These effects are concerned with the increase in appetite, hypersomnia, paralysis, interpersonal sensitiveness and mood reactiveness, which are found more among women. Increasingly, these effects lead towards the onset of depression at an earlier age, depressive episodes lasting longer, wide comorbidity range, more suicidal hazards and more functional disorders.

Shanahan et.al., (2015) proposed that the effect of depression is heavy over society, families and workplaces. Disorders related to depression are dominant and can be related to major damage upon life's quality of patients and the life of patient's relatives also suffers. The term known as major depressive disorder is related to the uneven mood, insomnia and anhedonia. Previous study concluded about the problems of sleep in order to develop the connection between sleep 
disorders and depression, as people with higher sleep disorders tends towards the phenomenon of pre-disposed that cause insomnia or sleep disturbance, which expose high risk of depression (Kalmbach et.al., 2015).

Cognitive and Affective/Somatic Factors of Depression: Depression has many factors such as behavioral, physiological and neurological. The factors which are concerned in assessing levels of depression are cognitive and affective/somatic. Cognitive symptoms are categorized as negative and include distorted thinking patterns, difficulty in concentrating, forgetfulness, distractibility and forgetfulness. Further symptoms include reduced reaction time, memory loss and indecisiveness (Tartakovsky, 2016). Affective and somatic factors of depression on the other hand includes symptoms such as frequent episodes of crying, irritability, anger, loss of interest in things that used to bring pleasure including sex. Beck divides these symptoms into two factors including cognitive and somatic/affective factors. He suggests that the somatic/affective symptoms of depression include loss of pleasure in daily activities, crying spells, feelings of agitation, loss of interest in daily activities, indecisiveness, loss of energy, changes in sleeping pattern, irritability in mood, changes in appetite, difficulty in concentration, tiredness or fatigue, and loss of interest in sex. Cognitive symptoms of depression on the other hand include sadness related thoughts, pessimism, thoughts about past failure, thoughts which brings guilty feelings, thoughts of punishment feelings, self-dislike, self-criticalness, suicidal thoughts or wishes and thoughts related to worthlessness (Beck, Steer, \& Brown, 1996).

Beck Depression Inventory-II (BDI-II): One of the most widely used depression measuring instruments is the Beck depression inventory. It can be used on many populations such as middle adolescents, late adolescents and adults, both male and female. Beck et.al., developed the Beck Depression Inventory Second Edition (BDIII) in 1996 in order to correspond for major depressive disorders with diagnostic criteria in the Diagnostic and Statistical Manual of Mental Disorders, Fourth Edition (DSM-IV) (Moore et.al., 2016). It is a selfreported inventory that measures depression in the form of its severity levels such as minimal, mild, moderate and severe. Here minimal level (scores between 0-13) indicates non-depressed population, mild level (scores between 14-19) indicates mildly depressed population, 
moderate level (scores between 20-28) indicates moderately depressed population and severe level (scores between 29-36) indicates severely depressed population. Items are further divided into cognitive and somatic/affective symptoms.(Beck, Steer, \& Brown, 1996).

Depression in Trainee Lady Health Visitor: Most of the student lady health visitors have high prevalence of stress in their life (Siddiqui et.al., 2020).It was observed internationally also that the increased work load of health workers increased mental stress and physical pressure, which made them prone to suffer from emotional stress (Rossouw, Seedat, Emsley \& Suliman, 2014). Past research states that health care facilitators, who are leading from the front, are always found to be loaded with multiple duties, which can prove to be alarming for their mental and physical health (Tao et.al., 2018).

Health workers suffer from anxiety and depression (Baloch, 2017). It is examined widely that the health workers experience various kind of stress during performing their duties (Kim et.al., 2014). Furthermore, $60 \%$ nurses are exposed to stress of at least average level and more (Nair et.al., 2018). Due to the above average stress they are unable to perform their duties and job with their full capacity (Brunie et.al., 2014).

Trainee lady health visitors are young and vulnerable strata of Pakistan's society due to their particular socioeconomic background, field job stress and patriarchal society which can lead to depression due to many external factors for example the life threatening situations created few years ago. According to Diwan 2013, lady health visitors have faced life-threatening situations from politico religious segments of society. This is a form of imported culture from across the border which has made its way in Pakistan's culture after the capturing of an Afghan leader from Pakistan in a series of attacks where on December 18, 2021, four polio workers were shot dead in different parts of Karachi. A day earlier a polio campaign volunteer in Karachi had been shot dead. Similar attacks were carried out on December 19, 2012, and in January 2013. These coordinated attacks resulted in a surge of fear among lady health visitors. Consequently, UN and WHO halted their polio vaccination campaigns. The attacks were thought to be planned by the followers of the captured leader as they suspected polio team members were involved in informing on his whereabouts about his residence). These incidents created an insecure and hopeless situation 
for the TLHVs. The fear they faced posed psychological stress and the LHVs have discussed these incidents when interviewed (Diwan 2013). Trainee lady health visitors face many problems in their work routine which includes sexual harassment, irregular wages, job insecurity, uncertain field conditions and life threatening situations imposed by fundamentalist and extremist religious factions of society and unhealthy patterns of patriarchal society, all the above stated issues may cause mental health problems like depression among intern trainee lady health visitors.

Rationale of the Study: The current study was planned to find prevalence of depression, its levels and its correlation with the somatic/affective and cognitive factors of depression among TLHVs in Pakistan. Knowledge about the prevalence of depression would help policy makers in designing policies for improving the mental health of trainee lady health visitors. A lady heath visitor with depressive symptoms wouldn't be able to fulfill her work demands as effectively as the one without it.

\section{HYPOTHESIS}

1) There would be a prevalence of depression among trainee lady health visitors in Pakistan.

2) There would be depression existing at different levels among trainee lady health visitors in Pakistan.

3) There would be a positive correlation between depression and somatic/affective factors of depression among trainee lady health visitors in Pakistan

4) There would be a positive correlation between depression and cognitive factors of depression among trainee lady health visitors in Pakistan.

\section{RESEARCH METHODOLOGY}

Sample: The study was carried out on a sample of 147 trainee lady health visitors who were 18-28 years of age, all of them had at least passed 12th grade (science group), they mostly belonged to lower socio-economic status, and had no physical disability or degenerative disease. The participants were selected through purposive sampling technique from different schools of public health in Karachi, Pakistan.

\section{Instrument}

Beck Depression Inventory-Second Edition (BDI-II): The Beck Depression Inventory-Second Edition (BDI-II) (Beck, Steer, \& 
Brown, 1996) is a 21-item self-reporting instrument for measuring severity of depression in different levels i.e. minimal, mild, moderate and severe. This version of BDI-II was developed for the assessment of symptoms for diagnosing depressive disorders listed in the American Psychiatric Association's Diagnostic and Statistical Manual of Mental Disorders-Fourth Edition (DSM-IV; 1994). Items are further divided into cognitive and somatic/affective symptoms. Item No. 4. Loss of Pleasure, 10. Crying, 11. Agitation, 12. Loss of Interest, 13. Indecisiveness, 15. Loss of Energy, 16. Changes in Sleeping Pattern, 17. Irritability, 18. Changes in Appetite, 19. Concentration Difficulty, 20. Tiredness or Fatigue, 21. Loss of Interest in Sex measures the somatic/affective symptoms of depression. whereas, item 1. Sadness 2. Pessimism, 3. Past Failure, 5. Guilty Feelings, 6. Punishment Feelings, 7. Self-Dislike, 8. Self-Criticalness, 9. Suicidal Thoughts or Wishes, 14. Worthlessness measures the cognitive symptoms of depression. Scoring BDI-II is easy by summing up ratings for the 21 items. Each item is rated on a 4-point scale ranging from 0 to 3 . The maximum total score is 63. Reliability of the BDI-II for outpatients was 0.92 (Beck, Steer, \& Brown, 1996).

Procedure: Purposive sampling technique was used for the selection of participants. The present study aimed to investigate the prevalence of depression and correlation between cognitive and somatic/affective symptoms of depression among trainee lady health visitors of Pakistan. For this purpose, trainee lady health visitors were approached at their workplace. Informed consent forms were provided for voluntary participation in which information about the purpose of study, goal, confidentiality, and privacy issues was included. They were asked to read it carefully before agreeing to participate in the study. Complete instructions were given to them about the study. Then demographic forms, consent forms along with BDI-II (Beck, Steer, \& Brown, 1996) were administrated on them. They were asked to fill these forms in a group setting of five to seven trainee lady health visitors. The researcher was present throughout when participants were completing their questionnaires. Participants were encouraged to answer every single question as there is no right or wrong answer. The administration process took five to ten minutes. After completing the questionnaires, the researcher thanked the participants. After collection of the data, the answer sheets were scored according to the instructions given in the respective questionnaire guideline. 


\section{RESULTS}

Descriptive statistics have been used to tabulate means and standard deviations of all the variables including prevalence of depression $(M=24.0, S D=10.01)$, the mean value and standard deviation of cognitive symptoms is $(M=8.97, S D=4.18)$, and somatic/affective symptoms $(M=15.03, S D=6.60)$ of trainee lady health visitors of Pakistan. Mean age 23 years Moreover, Pearson ' $r$ ' was used to determine the relationship between depression and somatic/affective factors of depression $(\mathrm{r}=.95, \mathrm{p}<0.01)$, and cognitive factors of depression $(\mathrm{r}=.88, \mathrm{p}<0.01)$.

TABLE-1

DESCRIPTIVE STATISTIC OF THE VARIABLES OF STUDY

\begin{tabular}{|l|l|l|l|l|l|l|l|}
\hline Variables & & $\boldsymbol{N}$ & $\boldsymbol{\%}$ & $\boldsymbol{M}$ & $\boldsymbol{S D}$ & $\begin{array}{l}\text { No.of } \\
\text { Items }\end{array}$ & $\begin{array}{l}\text { Cronbach's } \\
\text { Alpha }\end{array}$ \\
\hline Depression & Total & 147 & 100 & 24.01 & 10.01 & 21 & .78 \\
\hline & Minimal & 24 & 16.33 & & & & \\
\hline & Mild & 19 & 12.93 & & & & \\
\hline & Moderate & 57 & 38.77 & & & & \\
\hline & Severe & 47 & 31.97 & & & & \\
\hline
\end{tabular}

$N=$ Number of Participants; $M=$ Mean; $S D=$ Standard Deviation

TABLE-2

DESCRIPTIVE STATISTIC OF THE VARIABLES OF STUDY

\begin{tabular}{|l|l|l|l|l|l|}
\hline Variables & $\boldsymbol{N}$ & Min & Max & M & SD \\
\hline Somatic/ Affection & 147 & 1 & 30 & 15.03 & 6.60 \\
\hline Cognitive & 147 & 1 & 24 & 8.97 & 4.18 \\
\hline
\end{tabular}

$N=$ Number of participants; $M=$ Mean; $S D=$ Standard deviation

TABLE-3

RELATIONSHIP BETWEEN DEPRESSION AND ITS FACTORS BY USING BDI-II

\begin{tabular}{|c|c|c|}
\hline & & Overall depression \\
\hline \multirow{3}{*}{ Cog Factor } & Pearson Correlation & $.889 * *$ \\
\hline & Sig. (2-tailed) & .000 \\
\hline & $\mathbf{N}$ & 147 \\
\hline \multirow{3}{*}{ Somatic/affective } & Pearson Correlation & $.957^{* *}$ \\
\hline & Sig. (2-tailed) & .000 \\
\hline & $\mathbf{N}$ & 147 \\
\hline
\end{tabular}

**. Correlation is significant at the 0.01 level (2-tailed). 


\section{DISCUSSION}

The objectives of this study are to evaluate the prevalence of depression, prevalence of different levels of depression i.e. minimal, mild, moderate or severe in trainee lady health visitors of Pakistan, presence of correlation between depression and its factors namely somatic/affective and cognitive respectively.

TLHVs face many problems which may include sexual harassment, irregular wages, job insecurity, verbal abuse and the unhealthy patterns of a patriarchal society (Diwan, 2013). Health visitors experience vulnerability and sexual harassment by religious fundamentalists (Naqvi \& Khan, 2005). Trainee lady health visitors, in general, harbor concerns regarding removal or isolation from employment. It has also been found that health workers face verbal attacks and abuse by families that do not desire to vaccinate their child (Tsaras et.al., 2018). Along with the above-mentioned problems, the age, gender, socioeconomic status, and impact of the pressures of fundamentalist society contribute towards the development of depression among trainee lady health visitors.

The mean age of TLHVs participating in the study is $23, \mathrm{SD}=5$. This age is considered vulnerable for mental health as reported by Dr. Unaiza Niaz on women mental health (2004). Women as a female gender are twice more prone to be diagnosed with depression than men, (Mayo Clinic, n.d.). Lower socioeconomic status is linked with prevalence of depression (Hoebal et.al., 2017). The impact of the pressure of fundamentalist society also contributes towards the development of depression in lady health visitors due to physical attacks and casualties of lady health visitors in a series of attacks in 2012 (Diwan, 2013).

The first and second hypothesis of the study are about the prevalence of depression and different levels of depression in trainee lady health visitors of Pakistan. On average, a moderate level of depression was found in trainee lady health visitors with the mean value of $\mathrm{M}=24.02$. The current finding shows that $70.74 \%$ of the total sample suffers from moderate to severe depression, out of which $31.97 \%$ of them have severe clinical depression and $38.77 \%$ of the population of trainee lady health visitors are going through moderate depression. Study on health care visitors reveals that depression exist in health care workers due to long working hours and workplace stress (Pfefferbaum \& North, 2020). Moreover, it is little surprise that even 
after three decades from the launch of LHV program the objective is still not accomplished and the LHV program has not attained optimal functioning, this might be because of depression (Mumtaz, 2013). Studies on healthcare visitors reveals that they experience many psychological issues such as stress, burnout and depression. The high prevalence of depression is due to many reasons, while difference in levels of depression is because individuals respond to different situations according to their own genetic make-up and upbringing.

Trainee lady health visitors also experience occupational dysfunction such as hampered participation in daily activities, lack of choices in daily activities which are not in their control, failure in fulfilling inner needs on daily bases and imbalance in daily activities due to occupational burden (Akiyama \& Kyougoku, 2010; Miyake et al., 2014). Many researches are available on health care workers; however, literature on trainee lady health visitors in association with their mental health or even their physical health and occupational wellbeing is very limited, especially in Pakistan. A prior study conducted in Kenya also suggests that depression can develop in community health workers (Kiima et.al., 2009).

Unfortunately, limited literature is available on trainee lady health visitors in this regard. Many factors related to a job can lead towards job stress, such as salary delay, vague job description, field work, long hours (Invancevich, \& Matteson, 1980), and physical and sexual harassment at the workplace (Rospenda et.al., 2008). All these factors together or alone can lead towards many mental health issues such as anxiety, depression, low productivity, and job effectiveness, reduced motivation of workers, adjustment issues as well as negative effects on self-efficacy (Donald, \& DeCotiis, 1983; Spielberger, Vagg, $\&$ Wasala, 2003; Cherniss, 1980).

The third hypothesis of the research reveals the directional relationship between depression and somatic/affective factors of depression $(\mathrm{r}=0.95, \mathrm{p}<0.01)$ which indicates that there is a significantly strong positive relationship between depression and somatic/affective factors of depression in trainee lady health visitors of Pakistan. Current research reveals that in trainee lady health visitors somatic/affective are stronger than cognitive factors of depression. The high correlation with somatic/ affective factors is because in Pakistani society somatic and affective complaints are more attentively listened 
to than cognitive complaints. Moreover, people tend to narrate their problems with accompanying somatic and affective complaints.

The fourth hypothesis of the research also shows the directional relationship between depression and cognitive factors of depression $(\mathrm{r}=0.88, \mathrm{p}<0.01)$ which indicates that there is a significantly strong positive relationship between depression and cognitive factors of depression in trainee lady health visitors of Pakistan although its lesser than the affective/somatic. The cognitive model suggests that women are more prone towards having depression than men as a lot of cognitive variables play a role in developing depression i.e. the way women evaluate themselves, dealing with stressful situations and having negative ideation over self-concept (Girgus \& NolemHoeksema, 2006).

Many mental health issues have been the focus of previous studies internationally, however, limited amount of data is available in Pakistan with regards to trainee lady health visitors' mental health. Past research reveals that different levels of depression do exists in employees of different fields e.g., health care sectors and non-health care sectors in Australia (Sharpley et.al., 2019).

\section{CONCLUSION}

The main objective of this research was to explore the prevalence of depression in TLHVs and to find the correlation of depression with somatic/affective factor and cognitive factor of depression. The results found that moderate depression prevailed in trainee lady health visitors and this depression correlated with the somatic/affective factor of the depression. The somatic/affective factor of depression include: loss of pleasure, crying spells, agitation, loss of interest, indecisiveness, loss of energy, changes in sleeping pattern, irritability, changes in appetite, concentration difficulty, tiredness or fatigue and loss of interest in sex. TLHVs responses on these symptoms to express their depression is deep rooted in the setup of their Pakistan's socio-cultural background, where an emotion is expressed more easily in a somatic/affective manner rather than in a cognitive way. Excessive stressful factors born by TLHVs during their field work which included sexual harassment, character calling, rude behavior of people, irregular wages, lifethreatening situations, vulnerability and physical harassment along with risk factors of depression in TLHVs of Pakistan which include their gender, age, socioeconomic background and unhealthy patterns 
of Pakistan's patriarchal society has altogether caused symptoms of moderate depression in TLHVs.

The lady health worker appears destined to do her laborious job with her wellbeing remaining neglected. The job itself is very difficult to perform as it involves providing door-to-door visits, providing vaccinations to an uneducated and unwilling critical society. Therefore, it is little surprise that even after three decades from the launch of LHV program the objective is still not accomplished and the LHV program has not attained optimal functioning. A large number of women are still dying of complications in childbirth population is still on the rise and high infant mortality rate still exists. All this might be due to the presence of depression in TLHVs which eventually leads to depression leaving the lady health worker less effective in accomplishing her job goals. If societal, environment and cultural problems are coped well the levels of depression will automatically drop, resulting in a healthy, happy and energetic lady health visitor providing better information to the urban slums and uneducated rural areas of Pakistan.

\section{RECOMMENDATIONS AND FUTURE DIRECTION}

There are various suggestions for the future researchers. Firstly, it is conceivable to increase the size of the sample, in order to deliver an in-depth analysis about the importance of mental health of trainee lady health visitors. Secondly, researchers can replicate this study in different cultures to reveal the cultural factors involved in the development of depression in trainee lady health visitors. It will also help in policy making for trainee lady health visitors on governmental and private level to acknowledge the risks, related to mental health. It is primarily beneficial for the management to utilize useful interventions to support a healthier life at the workplace. It might be helpful in planning medical or psychological interventions for the betterment of mental health issues.

\section{REFERENCES}

Akiyama, E. \& Kyougoku, M. (2010). Examination of occupational dysfunction in workers: using occupational self assessment version2(OSA2). Sogo Rehabilitation, 38, 373-379.

American Psychological Association. (1994). Diagnostic and Statistical Manual of Mental Disorders ( $4^{\text {th }}$ ed.). Washington, DC: Author. 
Baloch, S. (2017). Lady health worker's problems highlighted, Dawn Retrived from: https://www.dawn.com/news/1311742

Beck, A.T., Steer, R.A., \& Brown, G.K. (1996). Beck Depression Inventory-II. San Antonio, 78(2), 490-498.

Brunie, A., Wamala-Mucheri, P., Otterness, C., Akol, A., Chen, M., Bufumbo, L., \& Weaver, M. (2014). Keeping community health workers in uganda motivated: Key challenges, facilitators, and preferred program inputs. Global Health: Science and Practice, 2(1), 103-116.

Chen, L.-C., Hsu, J.-W., Huang, K.-L., Bai, Y.-M., Su, T.-P., Li, C.-T., . . Tsai, S.-J. (2016). Risk of developing major depression and anxiety disorders among women with endometriosis: A longitudinal follow-up study. Journal of Affective Disorders, 190, 282-285.

Cherniss, C. (1980). Staff burnout-Job stress in the human services. National Criminal Justice Reference Service, 2, 191. Retrieved from: http://www.ncjrs.gov/App/publications/abstract.aspx?ID=75618

De Sousa, A. (2011). A freudian Theory and consciousness; A conceptual analysis. Mens Sana Monogr. 9(1), 210-217.

Diwan, M. (2013). Work experiences, professional identities and gender politics of Pakistan's lady health workers. (Dissertation). Queen's University: Canada

Donald, F. P., \& DeCotiis, T. A. (1983). Organizational determinants of job stress. Organizational Behavior and Human Performance, 32(2), 160-177.

Girgus, J., \& Nolen-Hoeksema, S. (2006). Cognition and depression. In Women and Depression: A Handbook for the Social, Behavioral, and Biomedical Sciences (pp. 147-175). Cambridge University Press. https://doi.org/10. 1017/CBO9780511841262.009

Hafeez, A., Mohamud, B.K., Shiekh, M.R., Shah, S.A.I., \& Jooma, R. (2011). Lady health workers programme in pakistan: Challenges, achievements and the way forward. JPMA: Journal of the Pakistan Medical Association, 61(3), 210.

Hassan, A. (2010). Participatory Development, pp.18-19, Orford University Press

Hoebel, J., Maske, U. E., Zeed, H., \& Lampert, T. (2017). Social inequalities and depressive symptoms in adults: th role of objective and subjective socioeconomic status. Plos One.

Invancevich, J. M., \& Matteson, M.T. (1980). Optimizing human resources: A case for preventive health and stress management. Organizational Dynamics, 9(2), 5-25.

Kalmbach, D.A., Pillai, V., Cheng, P., Arnedt, J.T., \& Drake, C.L. (2015). Shift work disorder, depression, and anxiety in the transition to rotating shifts: The role of sleep reactivity. Sleep Medicine, 16(12), 1532-1538. 
Kiima, D., Njenga, F., Shah, A., Okonji, M., Ayuyo, J., Baraza, M., Parker, E., \& Jenkins, E. (2009). Attitudes to depression among community health workers in Kenya. Epidemiologia e Psichiatria Sociale, 18, 4.

Kim, M.H., Mazenga, A.C., Devandra, A., Ahmed, S., Kazembe, P.N., Yu, X., . . . Sharp, C. (2014). Prevalence of depression and validation of the beck depression inventory-ii and the children's depression inventory-short amongst hiv-positive adolescents in malawi. Journal of the International AIDS Society, 17(1), 18965.

Depression in women: Understanding the gender gap. (n.d.). Mayo Clinic Staff.

Mumtaz, Z., Salway, S., Nykiforuk, C., Bhatti, A., Ataullahjan, A., \& Ayyalasomayajula, B. (2013). The role of social geography on lady health workers' mobility and effectiveness in Pakistan. Social Science \& Medicine, 91, 48-57.

Miyake, Y., Teraoka, M., Ogino, K., \& Kyougoku, M. (2014). Survey of the classification of occupational dysfunction among japanese rehabilitation therapists and the association of occupational dysfunction with job strain. Journal of Preventive Medicine, 9, 93-100.

Moore, A.A., Neale, M.C., Silberg, J.L., \& Verhulst, B. (2016). Substance use and depression symptomatology: Measurement invariance of the beck depression inventory (bdi-ii) among non-users and frequent-users of alcohol, nicotine and cannabis. PloS one, 11(4), e0152118.

Mumtaz, Z., Salway, S., Nykiforuk, C., Bhatti, A., Ataullahjan, A., \& Ayyalasomayajula, B. (2013). The role of social geography on lady health workers' mobility and effectiveness in pakistan. Social science \& medicine, 91, 48-57.

Nair, A., Turner, C., Heyman, I., Mataix-Cols, D., Lovell, K., Krebs, G., . . . O'Kearney, R. (2018). Moderators and predictors of outcomes in telephone delivered compared to face-to-face cognitive behaviour therapy for paediatric obsessive-compulsive disorder: Preliminary evidence from a non-inferiority rct. Cognitive behaviour therapy, 1-16.

Naqvi, H., \& Khan, M. M. (2005). Depression in primary care: difficulties and paradoxes. Journal-Pakistan Medical Association, 55(9), 393.

Peers for Progress (n.d.). Pakistan's lady health workers a national model for delivering primary healthcare and peer support. Retrieved from http://peersforprogress.org/wpcontent/uploads/2013/09/20130923_pakistans_lady_health_workers.pdf

Pfefferbaum, B., North, C.S. (2020). Mental health and the Covid-19 pandemic. N. Engl. J. Med, 383(6), 510-512.

Planning Commission Government of Pakistan, 1994

Pluskota, A. (2014). The application of positive psychology in the practice of eduation. Springerplus, 3, 147.

Porter, R., Bourke, C., Carter, J., Douglas, K., McIntosh, V., Jordan, J., . . . Frampton, C. (2016). No change in neuropsychological dysfunction or emotional processing during treatment of major depression with 
cognitive-behaviour therapy or schema therapy. Psychological medicine, 46(2), 393-404.

Reynolds, E.H. \& Wilson, J.V. (2013). Depression and anxiety in babylon. $J R$ Soc Med, 106(12), 478-481. Doi: 10.117/0141076813486262

Rospenda, K. M., Fujishiro, K., Shannon, C, A., \& Richman, J. A. (2008). Workplace harassment, stress, and drinking behavior overtime: Gender difference in a national sample. Addictive Behaviors, 33(7), 964-967.

Rossouw, L., Seedat, S., Emsley, R., \& Suliman, S. (2014). The prevalence of burnout and depression in medical doctors working in the cape town metropole community healthcare clinics and district hospitals of the provincial government of the western cape: A cross-sectional study. Official Journal of the South African Academy of Family Practice/Primary Care, 55(6), 567-573.

Sadler, P., McLaren, S., Klein, B., \& Jenkins, M. (2018). Advancing cognitive behaviour therapy for older adults with comorbid insomnia and depression. Cognitive behaviour therapy, 47(2), 139-154.

Schuch, J.J., Roest, A.M., Nolen, W.A., Penninx, B.W., \& De Jonge, P. (2014). Gender differences in major depressive disorder: Results from the netherlands study of depression and anxiety. Journal of affective disorders, 156, 156-163.

Shanahan, L., Zucker, N., Copeland, W.E., Bondy, C., Egger, H.L., \& Costello, E.J. (2015). Childhood somatic complaints predict generalized anxiety and depressive disorders during young adulthood in a community sample. Psychological medicine, 45(8), 1721-1730.

Sharpley, C. F., Bitsika, V., Sarmukadam, K., \& Agnew, L. L. (2019). Which kinds of work stress are related with which types of depression? Workplace satisfaction and subtypes of depression in rural Australians. Achives of Depression and Anxiety. 5(2), 034-041.

Siddiqui, S., Shah, N., Ayub, N., \& Khan, N. (2012). Student lady health visitors: their stress profile and willingness to practice. Journal of Ayub Medical College Abbottabad, 24, 3-4.

Spielberger, C. D., Vagg, P. R., \& Wasala, C. F. (2003). Occupational stress: job pressure and lack of support. In Quick, J. C., \& Tetrick, L. E.(Eds.). Handbook of Occupational Health Psychology. American Psychological Association. https://doi.org/10.1037/10474-009

Tabassum, N. (2016). Women in Pakistan status in socio-cultural and politicoLegal Domains. Higer Education Commission. http://prr.hec.gov.pk/jspui/handle/123456789//28

Tartakovsky, M. (2016). The cognitive symptoms of depression. PsychCentral Retrived from: https://psychcentral.com/lib/the-cognitive-symptoms-of-depression\#1

Tao, L., Guo, H., Liu, S., \& Li, J. (2018). Work stress and job satisfaction of community health nurses in southwest china. Biomedical Research, 29(3), $510-518$. 
Biannual Research Journal Grassroots Vol.55, No.II: 103-119

Tipton, C. M. (2014). The history of "Exercise is medicine" in ancient civilizations. Adv Physiol Educ. 38(2), 109-117. Doi: 10.1152/ advan.00136.2013

Tsaras, K., Papathanasiou, I. V., Vus, V., Panagiotopoulou, A., Katsou, M. A., Kelesi, M., \& Fradelos, E. C. (2018). Predicting Factors of Depression and Anxiety in Mental Health Nurses: A Quantitative Cross-Sectional Study. Medical Archives, 72(1), 62. 